\title{
Análise não linear estática de bloco sobre estaca baseada na Mecânica do Dano Contínuo
}

\section{Static nonlinear analysis of piles cap based on the Continuum Damage Mechanics}

\author{
Luiz Antonio Farani de Souza ${ }^{1}$
}

\section{Resumo}

O bloco sobre estacas é um importante elemento estrutural cuja função é transferir as ações da superestrutura para um grupo de estacas. A inspeção visual do comportamento do mesmo sob condições de serviço geralmente não é possível e, além disso, o conhecimento do seu verdadeiro desempenho estrutural é uma necessidade vital para a estabilidade global das construções. Neste artigo, uma análise não linear bidimensional é realizada, por meio do Método dos Elementos Finitos, de um bloco rígido de concreto armado sobre duas estacas encontrado na literatura. Adota-se para o material concreto um modelo constitutivo baseado na Mecânica do Dano Contínuo, com a possibilidade de prever um comportamento diferenciado à tração e à compressão. $\mathrm{O}$ aço é descrito por um modelo elastoplástico bilinear. A trajetória de equilíbrio é obtida com a técnica de iteração Comprimento de Arco em associação com o Método de Newton - Raphson. Os resultados numéricos obtidos com o código computacional desenvolvido são confrontados com os resultados experimentais e numéricos disponíveis e com a solução analítica, e têm o objetivo de avaliar a potencialidade da modelagem proposta como uma ferramenta numérica de investigação quanto à determinação da força de ruptura e a distribuição do dano no bloco.

Palavras-chave: Bloco sobre estacas. Concreto. Mecânica do Dano. Elementos Finitos.

\begin{abstract}
The piles cap is an important structural element whose function is to transfer the actions of the superstructure for a group of piles. The visual inspection of the piles cap behavior under service conditions is not possible and, in addition, the knowledge of its actual structural performance is a vital necessity for the constructions overall stability. In this paper, a two-dimensional nonlinear analysis is carried out, by means of Finite Element Method, of a reinforced concrete pile caps with two piles found in the literature. It adopts for the material concrete a constitutive model based on the Continuum Damage Mechanics, with the possibility to provide a tensile and compression differentiated behavior. The steel is described by an elastoplastic bilinear model. The equilibrium path is achieved by Arc Length iteration technique in association with the Newton - Raphson Method. The numerical results obtained with the developed computational code are compared with the available experimental and numerical results and the analytical solution, and have the objective of evaluate the potential of the proposed modeling as an investigation numerical tool to determine the rupture force and the damage distribution in the piles cap.
\end{abstract}

Keywords: Piles cap. Concrete. Damage Mechanics. Finite Elements.

${ }^{1}$ Docente do Curso de Engenharia Civil da Universidade Tecnológica Federal do Paraná - UTFPR; lasouza@utfpr.edu.br. 


\section{Introdução}

Os blocos sobre estacas são elementos estruturais de fundação cuja finalidade é transmitir às estacas as ações oriundas da superestrutura. $\mathrm{O}$ número de estacas no bloco depende dos esforços solicitantes na base do pilar, da capacidade geotécnica do solo e das condições disponíveis de construção, possibilitando a definição do tipo de estaca e a determinação de sua capacidade portante (OLIVEIRA et al., 2014).

Os processos correntes para o dimensionamento do bloco sobre estacas não fornecem ao engenheiro de estruturas um claro entendimento acerca do comportamento físico desse elemento. Em geral, a inspeção visual do mesmo sob condições de serviço não é possível e, além disso, como o comportamento adequado dessa estrutura é uma necessidade vital para a estabilidade global das construções, o conhecimento do seu verdadeiro desempenho torna-se de fundamental importância (SOUZA; BITTENCOURT, 2004).

$\mathrm{O}$ avanço das técnicas computacionais e do Método dos Elementos Finitos (MEF) possibilitou analisar resultados de ensaios experimentais por meio de simulação numérica. Neste artigo, fazse uma análise não linear estática bidimensional do comportamento estrutural de um bloco rígido de concreto armado sobre duas estacas ensaiado experimentalmente por Mautoni (1972). As simulações numéricas são realizadas com 0 programa livre Scilab.

A trajetória de equilíbrio é obtida considerando a estratégia de iteração Comprimento de Arco associada com o método de Newton - Raphson. Como critério de convergência no final das iterações para cada passo de carga, adota-se um controle concomitante de força e deslocamento.

Para o material concreto emprega-se um modelo constitutivo baseado na Mecânica do Dano Contínuo proposto por Mazars (1984), com a possibilidade de prever um comportamento diferenciado à tração e à compressão. $\mathrm{O}$ aço é descrito por um modelo elastoplástico bilinear.

$\mathrm{Na}$ malha de Elementos Finitos as armaduras longitudinais são simuladas por elementos de treliça plana introduzidos como linhas de material mais rígido no modelo estrutural, e o concreto é discretizado por elementos quadrangulares lineares isoparamétricos.

Os resultados numéricos obtidos com o código computacional desenvolvido são confrontados com o experimental, com o modelo numérico apresentado por Souza (2004) e com a solução analítica obtida por meio do modelo de Escoras e Tirantes. A comparação dos resultados tem o objetivo de avaliar a potencialidade da modelagem proposta como uma ferramenta numérica de investigação quanto à determinação da força de ruptura e a distribuição do dano no bloco. Em adição, contribui para comprovar os fundamentos teóricos dos blocos sobre estacas.

\section{Modelos constitutivos dos materiais}

Uma representação consistente do comportamento mecânico dos materiais envolvidos na análise estrutural é de grande importância para o bom desempenho da simulação numérica por meio do MEF. Assim, modelos matemáticos devem ser estabelecidos para o desenvolvimento das relações constitutivas dos materiais (leis tensão-deformação). No decorrer desta seção são descritos os modelos constitutivos adotados para o concreto e o aço.

\section{Concreto}

O concreto apresenta, em relação aos modos de ruptura, um comportamento distinto à tração e à compressão. A ruptura do concreto por esforços de tração é ocasionada pela formação de fissuras e consequente perda de resistência normal à direção da fissura. Quanto à ruína na compressão, o concreto apresenta um comportamento que pode ser considerado como plástico, que é o esmagamento ocasionado pela superação 
da coesão interna por efeito da tensão de cisalhamento caracterizada por grande quantidade de microfissuras (PITUBA; PROENÇA, 2005).

Neste modelo, proposto por Mazars (1984), supõe-se que o dano se inicia quando a deformação equivalente atinge um valor de deformação de referência $\varepsilon_{\mathrm{d} 0}$, determinado em ensaios de tração uniaxial em correspondência à tensão máxima. A relação constitutiva é dada por (PITUBA; PROENÇA, 2005):

$$
\sigma=(\mathrm{I}-\mathrm{dI}) \mathrm{C}_{0} \varepsilon
$$

sendo $\mathbf{I}$ o tensor identidade, $\mathbf{C}_{0}$ o tensor constitutivo do material íntegro, $\mathrm{d}$ o escalar dano, $\varepsilon$ o tensor de deformações, e $\sigma$ o tensor de tensões. No caso do modelo unidimensional, a deformação equivalente $\tilde{\varepsilon}$ é avaliada neste trabalho pela seguinte equação:

$$
\tilde{\varepsilon}=\sqrt{\varepsilon_{1}^{2}+\varepsilon_{2}^{2}}
$$

sendo $\varepsilon_{\mathrm{i}}, \mathrm{i}=1,2$, as componentes de deformação principal. Considerando-se um carregamento continuamente crescente ou radial, das curvas tensão-deformação obtidas em ensaios uniaxiais de tração e compressão, podem ser determinadas explicitamente as variáveis de dano $d_{T}$ e $d_{C}$ da seguinte forma:

$$
\begin{aligned}
& \mathrm{d}_{\mathrm{T}}(\tilde{\varepsilon})=1-\frac{\varepsilon_{\mathrm{d} 0}\left(1-\mathrm{A}_{\mathrm{T}}\right)}{\widetilde{\varepsilon}}-\frac{\mathrm{A}_{\mathrm{T}}}{\mathrm{e}^{\left(\mathrm{B}_{\mathrm{T}} \tilde{\varepsilon}-\varepsilon_{\mathrm{d} 0}\right)}} \\
& \mathrm{d}_{\mathrm{C}}(\tilde{\varepsilon})=1-\frac{\varepsilon_{\mathrm{d} 0}\left(1-\mathrm{A}_{\mathrm{C}}\right)}{\widetilde{\varepsilon}}-\frac{\mathrm{A}_{\mathrm{C}}}{\mathrm{e}^{\left(\mathrm{B}_{\mathrm{C}} \tilde{\varepsilon}-\varepsilon_{\mathrm{d} 0}\right)}}
\end{aligned}
$$

sendo $A_{T}$ e $B_{T}$ parâmetros característicos do material em tração uniaxial, $A_{C}$ e $B_{C}$ parâmetros do material em compressão uniaxial, e $\varepsilon_{\mathrm{d} 0}$ a deformação específica elástica limite. Os subíndices "T" e "C" significam tração e compressão, respectivamente. As variáveis de dano $\left(d_{T}\right.$ e $\left.d_{C}\right)$ foram avaliadas separadamente para cada ponto de Gauss na integração numérica (obtenção do vetor de força interna) conforme as Equações (3) e (4), não se considerando a combinação das mesmas.
$A c ̧ o$

Utiliza-se um modelo uniaxial para descrever o comportamento das armaduras, uma vez que em estruturas de concreto armado as barras de aço resistem, fundamentalmente, a esforços axiais. O comportamento do aço é representado por um modelo elastoplástico bilinear, supondo o mesmo comportamento à tração e à compressão. Assim, a tensão no aço é determinada por:

$$
\sigma= \begin{cases}\mathrm{E}_{\mathrm{s}} \varepsilon, & \text {, se } \varepsilon<\mathrm{B}_{1} \frac{\sigma_{\mathrm{y}}}{\mathrm{E}_{\mathrm{s}}} \\ \mathrm{E}_{\mathrm{st}} \varepsilon & \text {, se } \varepsilon \geq \mathrm{B}_{1} \frac{\sigma_{\mathrm{y}}}{\mathrm{E}_{\mathrm{s}}}\end{cases}
$$

sendo $\mathrm{E}_{\mathrm{s}} \mathrm{o}$ módulo de elasticidade longitudinal inicial do aço, $\sigma_{\mathrm{Y}}$ a tensão de escoamento, $\mathrm{E}_{\mathrm{st}}=\mathrm{k}_{\mathrm{s}}$ $\mathrm{E}_{\mathrm{s}} \mathrm{o}$ módulo de elasticidade longitudinal após o escoamento do aço, e $\mathrm{B}_{1}$ e $\mathrm{k}_{\mathrm{s}}$ constantes do modelo.

\section{Método de solução}

Para problemas de ponto limite, ao aplicar o método de Newton - Raphson com controle de força, a matriz de rigidez tende a singularizar nas proximidades desse ponto em sua trajetória ascendente. Uma alternativa para detectar e ultrapassar o ponto limite é a utilização de métodos de solução associados ao método de Newton - Raphson, como por exemplo, o método de Comprimento de Arco.

O método de Comprimento de Arco caracteriza-se por apresentar um controle concomitante de força e deslocamento. Há duas incógnitas: o incremento do fator de força $\Delta \varphi \mathrm{e}$ o vetor de incremento de deslocamento $\Delta \mathbf{u}$. Em cada passo de solução, as trajetórias de iteração são perpendiculares aos arcos, que por sua vez podem ser aproximados por tangentes à trajetória de equilíbrio (RAMM, 1981). Considerando a técnica de Comprimento de Arco com o método Newton - Raphson, as equações de equilíbrio para a i - ésima iteração podem ser escritas como:

$$
\mathbf{K}_{\mathrm{T}} \Delta \mathbf{u}^{\mathrm{i}}=\Delta \varphi^{\mathrm{i}} \mathbf{R}_{\mathbf{0}}+\Delta \mathbf{Q}^{\mathrm{i}-\mathbf{1}}
$$


sendo $\Delta \varphi^{\mathrm{i}}$ o incremento do fator de força da iteração i, $\Delta \mathbf{u}^{\mathrm{i}}$ o vetor incremento de deslocamento, $\mathbf{R}_{\mathbf{0}}$ o vetor de forças de referência, $\mathbf{K}_{\mathrm{T}}$ a matriz de rigidez tangente atualizada a cada iteração, e $\Delta \mathbf{Q}^{\mathbf{i}-1}$ o vetor de forças não equilibradas dado por:

$$
\Delta Q^{i-1}=R_{e x t}^{i-1}-F_{i n t}^{i}
$$

sendo $\mathbf{R}_{\text {ext }}{ }^{\mathrm{i}-1}$ o vetor de forças externas, e $\mathbf{F}_{\text {int }}{ }^{\mathbf{i}}$ o vetor de forças internas. $O$ vetor $\mathbf{R}_{\text {ext }}{ }^{\mathrm{i}-1}$ deve ser escrito em função do fator de força $\varphi^{\mathrm{i}-1}$, atualizado ao final da iteração anterior e do vetor de forças de referência $\mathbf{R}_{\mathbf{0}}$, constante, por meio da seguinte relação:

$$
\mathbf{R}_{\text {ext }}{ }^{i-1}=\varphi^{i-1} \mathbf{R}_{0}
$$

Para um sistema de ordem $n+1$, equivalendo $\mathrm{n}$ ao número de graus de liberdade da estrutura, tem-se:

$$
\left[\begin{array}{cc}
\mathbf{K}_{\mathrm{T}} & -\mathbf{R}_{0} \\
\Delta \mathbf{u}^{1} & \Delta \varphi^{1}
\end{array}\right]\left\{\begin{array}{c}
\Delta \mathbf{u}^{\mathrm{i}} \\
\Delta \varphi^{\mathrm{i}}
\end{array}\right\}=\left\{\begin{array}{c}
\Delta \mathbf{Q}^{\mathrm{i}-1} \\
0
\end{array}\right\}
$$

sendo $\Delta \mathbf{u}^{1}$ o primeiro vetor de incrementos de deslocamento do passo de solução, e $\Delta \varphi^{1}$ o primeiro incremento do fator de força no referido passo. Nota-se que a resolução do sistema dado em (9) gera um sistema de equações com solução não trivial mesmo que a matriz $\mathbf{K}_{\mathrm{T}}$ seja singular, o que representa grande vantagem para a solução de problemas com ponto limite.

Entretanto, há o problema da matriz de rigidez não ser simétrica. A fim de contornar esse problema, Wessels (1977) propôs uma alternativa que consiste em dividir o vetor de incremento de deslocamentos $\Delta \mathbf{u}^{\mathrm{i}}$ em duas parcelas: $\Delta \mathbf{u}_{\mathrm{Q}}^{\mathrm{i}}$ e $\Delta \mathbf{u}_{{ }_{\mathrm{R}}}^{\mathrm{i}}$. Essas parcelas são obtidas pela resolução dos sistemas:

$$
\begin{aligned}
& \mathrm{K}_{\mathrm{T}} \Delta \mathrm{u}^{\mathrm{i}}{ }_{\mathrm{Q}}=\Delta \mathrm{Q}^{\mathrm{i}-\mathbf{1}} \\
& \mathrm{K}_{\mathrm{T}} \Delta \mathbf{u}^{\mathrm{i}}{ }_{\mathrm{R}}=\mathrm{R}_{\mathbf{0}}
\end{aligned}
$$

$\mathrm{O}$ incremento do fator de força é calculado por:

$$
\Delta \varphi^{\mathrm{i}}=-\frac{\Delta \mathbf{u}^{1}: \Delta \mathbf{u}^{\mathrm{i}}{ }_{\mathrm{Q}}}{\Delta \mathbf{u}^{1}: \Delta \mathbf{u}^{\mathrm{i}}{ }_{\mathrm{R}}+\Delta \varphi^{1}}
$$

Com o objetivo de limitar os processos iterativos, dois critérios de convergência são estabelecidos: um para os deslocamentos e outro para as forças.

O critério de convergência para os deslocamentos deve obedecer a seguinte desigualdade:

$$
\frac{\left\|\Delta u^{i}\right\|}{\left\|u^{i}\right\|} \leq u_{\text {tol }}
$$

sendo o numerador a norma euclidiana do vetor de incremento de deslocamento $\Delta \mathbf{u}^{\mathrm{i}}$ correspondente à iteração i, e o denominador a norma euclidiana do vetor de deslocamento total $\mathbf{u}^{\mathbf{i}}=\mathbf{u}^{\mathrm{i}-1}+\Delta \mathbf{u}^{\mathrm{i}}$ da i - ésima iteração.

O critério de convergência para forças deve obedecer à relação:

$$
\frac{\left\|\Delta Q^{i}\right\|}{\left\|\varphi^{\mathrm{i}} \mathbf{R}_{0}\right\|} \leq \mathbf{Q}_{\text {tol }}
$$

sendo o numerador a norma euclidiana do incremento de força não equilibrada $\Delta \mathbf{Q}^{\mathbf{i}}$ correspondente à iteração $\mathrm{i}$, e o denominador a norma euclidiana do incremento de força do passo de solução.

O sinal do incremento do fator de força pode ser positivo ou negativo na primeira iteração de cada passo de carga. Uma técnica que avalie corretamente esse sinal é necessária de maneira a alterar o sentido de crescimento da força e, consequentemente, ultrapassar um ponto limite da trajetória de equilíbrio. Há procedimentos para avaliar o sinal desse incremento como, por exemplo, os propostos por Krenk e Hededal (1995) e Yang e Kuo (1994). No método de solução utilizado neste trabalho não foi implementada nenhuma técnica.

Modelo analítico para o cálculo da força de ruptura do bloco sobre duas estacas

O dimensionamento de blocos rígidos com o Método das Bielas, baseado no trabalho experimental realizado por Blévot e Frémy (1967), consiste em idealizar no interior da estrutura real 
uma treliça tridimensional formada por escoras de concreto e tirantes de aço que se encontram em zonas denominadas de regiões nodais. O modelo desenvolvido dessa maneira é chamado de Modelo de Escoras e Tirantes e, para a garantia de ductilidade, deve-se sempre garantir que os tirantes de aço vão escoar antes das escoras e das regiões nodais entrarem em ruína (SOUZA; BITTENCOURT, 2004).

O nível de tensão estabelecido nas regiões nodais, bem como nas escoras, deve ser limitado a certa parcela da resistência à compressão do concreto, de maneira a evitar fissurações indesejáveis e até mesmo uma possibilidade de ruína prematura (SOUZA; BITTENCOURT, 2005).

No projeto de blocos sobre duas estacas considera-se o esquema de forças internas mostrado na Figura 1, sendo que a treliça tem a barra tracionada localizada logo acima da cota de arrasamento das estacas representada pela força $\mathrm{R}_{\mathrm{st}}$, e as diagonais comprimidas são inclinadas e representadas pela força $R_{c b}$.

Figura 1 - Modelo de cálculo para blocos sobre duas estacas.
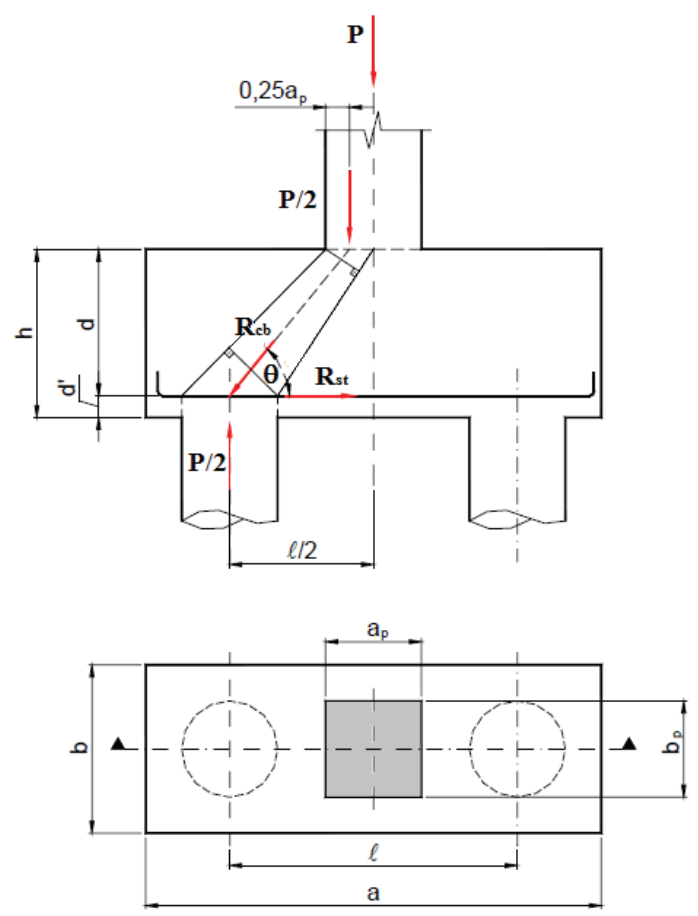

Fonte: adaptada de Munhoz (2004).
A força de tração $\mathrm{R}_{\mathrm{st}}$ nas barras da armadura é determinada pela seguinte equação (MUNHOZ, 2004):

$$
R_{s t}=\frac{P\left(2 l-a_{p}\right)}{8 d}
$$

sendo 1 o espaçamento entre os eixos das estacas, $\mathrm{d}$ a altura útil do bloco, e $\mathrm{a}_{\mathrm{p}}$ a dimensão do pilar.

Segundo Souza e Bittencourt (2004), a força $R_{\text {st }}$ pode ser obtida por:

$$
\mathbf{R}_{\mathrm{st}}=\frac{\mathbf{P}\left(2 \mathrm{l}-\mathrm{a}_{\mathrm{p}}\right)}{8 \mathrm{~d}}=\mathrm{A}_{\mathrm{s}} \mathrm{f}_{\mathrm{yk}}
$$

sendo $\mathrm{A}_{\mathrm{s}}$ a área da seção transversal das barras de aço, e $f_{y k}$ a resistência característica ao escoamento do aço. Esses autores recomendam, baseado no trabalho de Moraes (1976), que as tensões máximas de compressão no concreto - na escora junto ao pilar $\left(\sigma_{c b p}\right)$ e na escora junto às estacas $\left(\sigma_{c b e}\right)$ - sejam limitadas aos valores apresentados nas seguintes inequações, respectivamente:

$$
\begin{aligned}
\sigma_{\mathrm{cbp}} & =\frac{P}{\mathrm{~A}_{\mathrm{p}} \operatorname{sen}^{2}(\theta)} \leq 1,4 \mathrm{f}_{\mathrm{ck}} \\
\sigma_{\mathrm{cbe}} & =\frac{P}{2 \mathrm{~A}_{\mathrm{e}} \operatorname{sen}^{2}(\theta)} \leq 1,4 \mathrm{f}_{\mathrm{ck}}
\end{aligned}
$$

sendo $f_{c k}$ a resistência característica à compressão do concreto, $\mathrm{A}_{\mathrm{p}}$ a área da seção transversal do pilar, $\mathrm{A}_{\mathrm{e}} \mathrm{a}$ área da seção transversal da estaca, e $\theta$ o ângulo de inclinação das escoras determinado por:

$$
\theta=\operatorname{tg}^{-1}\left(\frac{d}{\frac{1}{2}-\frac{a_{p}}{4}}\right)
$$

A força de ruptura analítica para o bloco sobre duas estacas é calculada pelo menor valor absoluto de P obtido das Equações (16) a (18).

\section{Simulação numérica}

Este problema consiste de um bloco rígido de concreto armado sobre duas estacas ensaiado por Mautoni (1972). Este autor realizou vários ensaios de blocos induzindo-os a romperem por corte, por meio da disponibilização de uma taxa de armadura muito superior àquela apontada pelo Método das 
Bielas. A análise não linear deste problema consiste em avaliar a potencialidade da modelagem proposta como uma ferramenta numérica de investigação quanto à força de ruptura e a distribuição do dano no concreto.

O bloco em questão possui largura $0,15 \mathrm{~m} \mathrm{e}$ altura $0,25 \mathrm{~m}$, o pilar tem comprimento $0,15 \mathrm{~m}$ e as estacas têm comprimento $0,1 \mathrm{~m}$. A distância entre os eixos das estacas é $0,32 \mathrm{~m}$ e a saliência entre a face interna da estaca e a face do pilar é $0,09 \mathrm{~m}$. $\mathrm{Na}$ Figura 2 é apresentado um desenho esquemático da configuração geométrica do bloco. A armadura longitudinal é constituída de seis barras com diâmetro 12,5 mm. Os parâmetros dos modelos constitutivos dos materiais aço e concreto são apresentados na Tabela 1. Considerou-se aderência perfeita entre as armaduras longitudinais e o concreto.

Figura 2 - Bloco rígido sobre duas estacas.

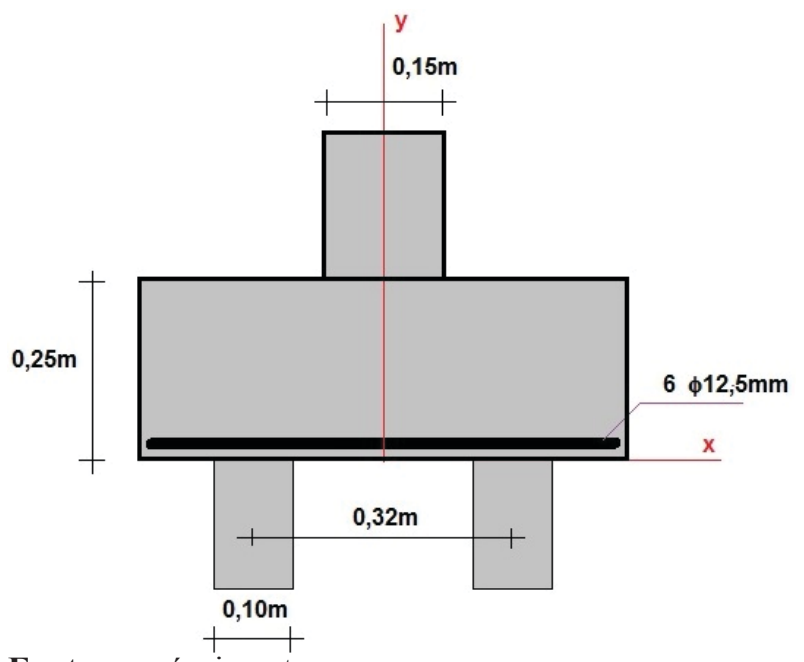

Fonte: o próprio autor.

Dada à simetria de carregamento e geometria, discretizou-se por Elementos Finitos apenas metade da estrutura. Na simulação numérica, utilizou-se uma malha de 217 nós, sendo o concreto simulado por 180 elementos quadrangulares lineares isoparamétricos (dois graus de liberdade/nó), e a armadura longitudinal por 30 elementos de treliça plana (dois graus de liberdade/nó) introduzidos como linhas de material mais rígido no modelo estrutural.
No modelo bidimensional o pilar e a estaca não foram discretizados, sendo o efeito desses elementos substituídos por condições de contorno: restrição de translação na direção $\mathrm{x}$ na região destinada ao pilar, restrição de translação na direção y na região destinada à estaca, e restrições de translação nas direções x e y no nó localizado na face interna da estaca. A força $\mathrm{P}$ foi distribuída nos nós da malha na região onde está localizado o pilar.

Tabela 1 - Propriedades materiais.

\begin{tabular}{cc}
\hline Concreto & Aço \\
\hline $\mathrm{E}_{\mathrm{c} 0}=33110,73 \mathrm{MPa}$ & $\mathrm{E}_{\mathrm{s}}=210 \mathrm{GPa}$ \\
$\nu=0,2$ & $\mathrm{k}_{\mathrm{s}}=0,1$ \\
$\varepsilon_{\mathrm{d} 0}=5,010^{-5}$ & $\sigma_{\mathrm{Y}}=720 \mathrm{MPa}$ \\
$\mathrm{A}_{\mathrm{T}}=0,9$ & $\mathrm{~B}_{1}=1$ \\
$\mathrm{~B}_{\mathrm{T}}=1435$ & \\
$\mathrm{~A}_{\mathrm{C}}=0,9$ & \\
$\mathrm{~B}_{\mathrm{C}}=1435$ & \\
\hline
\end{tabular}

Fonte: o próprio autor.

Supôs-se o Estado Plano de Tensões e os erros máximos admitidos para o deslocamento e a força durante a simulação no final de cada incremento de força foram $\mathrm{u}_{\mathrm{tol}}=10^{-2} \mathrm{e} \mathrm{Q}_{\mathrm{tol}}=10^{-2}$, respectivamente.

Na Figura 3 são apresentadas as curvas numéricas deslocamento vertical no eixo de simetria do bloco versus força, obtidas da modelagem proposta e do modelo tridimensional proposto por Souza (2004). A distribuição do dano $d$ no concreto na metade do bloco é mostrada na Figura 4. Na Figura 5 é apresentado o panorama de fissuração do bloco para a força $\mathrm{P}$ igual a $780 \mathrm{kN}$ referente ao ensaio realizado por Mautoni (1972).

Simulações com o modelo de dano de Mazars (1984) podem conduzir a deformações excessivas na estrutura a partir de um determinado incremento de força (GUELLO; BITTENCOURT, 2002). Para limitar essas deformações, considerou-se durante a análise que o valor máximo da variável dano fosse igual a 0,79 , isto é, $\mathrm{d}_{\mathrm{i}} \leq 0,79, \mathrm{i}=\mathrm{C}, \mathrm{T}$. Um aspecto importante a ser evidenciado no modelo de Mazars é que os resultados são sensíveis quando se varia o valor de $\varepsilon_{\mathrm{d} 0}$, uma vez que altera o ponto 
de surgimento do dano e, consequentemente, o mapa da distribuição do mesmo na peça (SOUZA; MACHADO, 2013).

Figura 3 - Deslocamento vertical no eixo de simetria do bloco versus força.

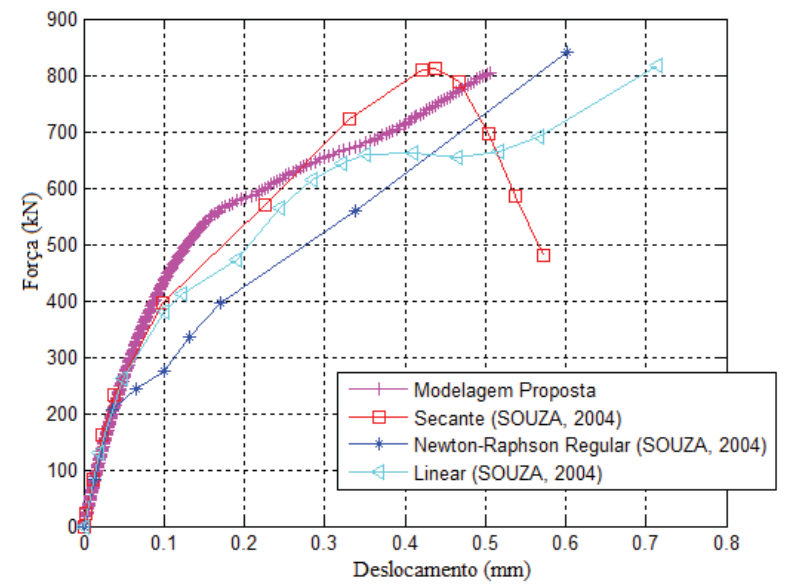

Fonte: o próprio autor.

Figura 4 - Distribuição do dano no concreto na metade do bloco sobre duas estacas

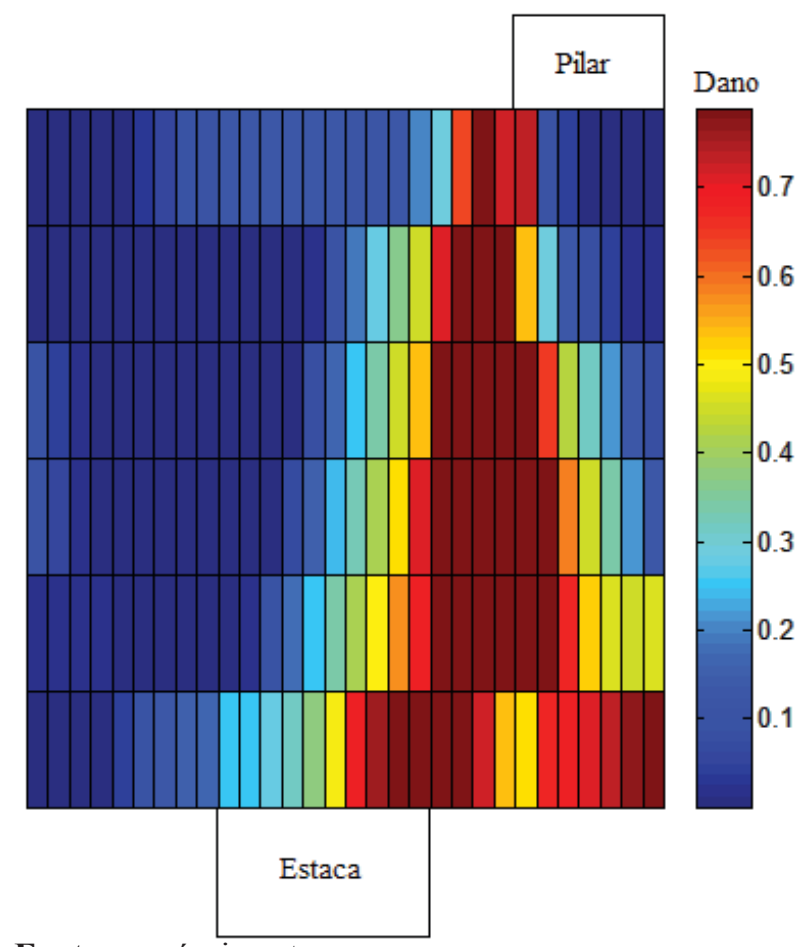

Fonte: o próprio autor.

Fazendo-se a análise da curva obtida da modelagem proposta na Figura 3, observa-se que o bloco entrou em ruína para a força $\mathrm{P}$ igual a
$805,0023 \mathrm{kN}$, aproximadamente, ficando próxima da força de ruptura experimental $(800,0 \mathrm{kN})$.

Conforme a distribuição do dano no bloco na Figura 4, houve o desenvolvimento de um plano de ruptura principal, definido pelos elementos mais escuros (d 0,79), que se estendem da face interna da estaca até a face lateral do pilar. Dessa forma, o modo de ruptura do bloco sobre estacas se deu por corte, ficando de acordo com os resultados experimentais.

Figura 5 - Panorama de fissuração do bloco sobre duas estacas para a força de $780 \mathrm{kN}$.

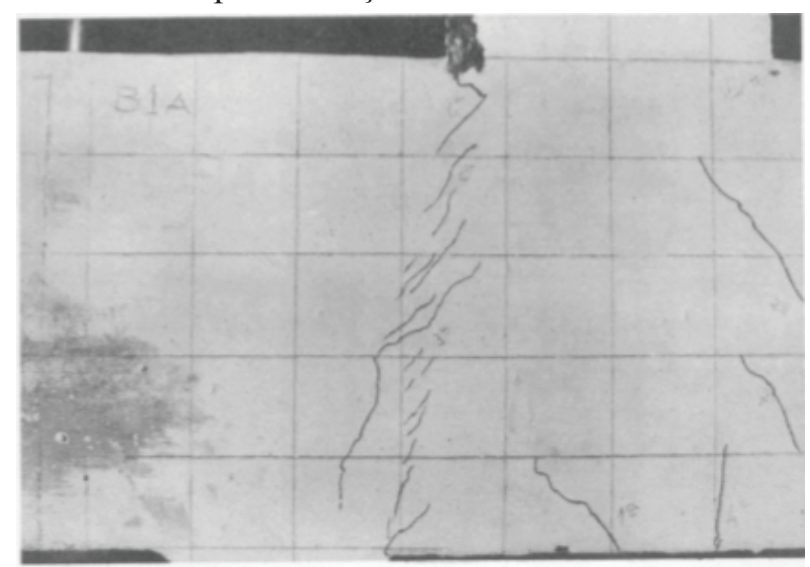

Fonte: Mautoni (1972).

A tensão máxima na direção $\mathrm{x}$ desenvolvida para as armaduras foi cerca de 486,32 $\mathrm{MPa}$, ficando relativamente distante do limite de escoamento de $720 \mathrm{MPa}$. Assim, pode-se concluir que efetivamente a ruína da estrutura se deu pelo desenvolvimento das fissuras geradas entre a estaca e o pilar.

Ao comparar as curvas preditas pelos modelos na Figura 3, verificam-se diferenças nas respostas numéricas que são explicadas, em parte, por: dimensionalidade, metodologia de solução das equações não lineares, modelos constitutivos para o concreto e o aço, e critério de convergência. Nessa figura, as curvas numéricas apresentadas por Souza (2004) foram obtidas com o programa DIANA por meio de um modelo tridimensional constituído de elementos do tipo CHX60, que é um elemento sólido (brick) isoparamétrico de 20 nós dotado de 
interpolação quadrática e integração de Gauss, e utilizou-se nas simulações numéricas o modelo de fissuração distribuída do tipo Fixed Crack Model. Segundo esse autor, os resultados dessas análises apontaram o "Método Secante" como sendo o melhor processo de solução para a investigação conduzida e, além disso, a força de ruína foi de $812,0 \mathrm{kN}$.

$\mathrm{Na}$ determinação da força de ruptura analítica (P) para o bloco rígido são considerados, conforme Souza (2004), os valores dos parâmetros apresentados na Tabela 2. Na Tabela 3 são mostrados os valores da força e do modo de ruptura da estrutura preditos pelos modelos numéricos e pelo analítico em comparação com os experimentais.

Tabela 2 - Parâmetros do modelo analítico.

\begin{tabular}{cc}
\hline Parâmetro & Valor \\
\hline $\mathrm{A}_{\mathrm{p}}$ & $225,00 \mathrm{~cm}^{2}$ \\
$\mathrm{~A}_{\mathrm{e}}$ & $150,00 \mathrm{~cm}^{2}$ \\
$\mathrm{~A}_{\mathrm{s}}$ & $7,32 \mathrm{~cm}^{2}$ \\
$\mathrm{f}_{\mathrm{ck}}$ & $2,83 \mathrm{kN} / \mathrm{cm}^{2}$ \\
$\mathrm{f}_{\mathrm{yk}}$ & $72,00 \mathrm{kN} / \mathrm{cm}^{2}$ \\
$\theta$ & $59,74^{\circ}$ \\
$\mathrm{d}$ & $21,00 \mathrm{~cm}$ \\
$\mathrm{a}_{\mathrm{p}}$ & $15,00 \mathrm{~cm}$ \\
$\mathrm{l}$ & $32,00 \mathrm{~cm}$ \\
\hline
\end{tabular}

Fonte: o próprio autor.

Tabela 3 - Valores da força de ruptura (P) e o modo de ruptura obtidos a partir dos modelos numéricos e analítico e do ensaio experimental.

\begin{tabular}{ccc}
\hline Método & $\begin{array}{c}\text { Força de ruptura } \\
(\mathbf{k N})\end{array}$ & $\begin{array}{c}\text { Modo de } \\
\text { ruptura }\end{array}$ \\
\hline $\begin{array}{c}\text { Numérico } \\
\text { (modelagem proposta) }\end{array}$ & 805,0023 & Corte \\
$\begin{array}{c}\text { Numérico } \\
\text { (SOUZA, 2004) }\end{array}$ & 812,00 & Corte \\
Modelo Analítico & 665,07 & Corte \\
$\begin{array}{c}\text { Experimental } \\
\text { (MAUTONI, 1972) }\end{array}$ & 800,00 & Corte \\
\hline
\end{tabular}

Fonte: o próprio autor.
Vê-se na Tabela 3 que os modelos conseguiram predizer o tipo de falha do bloco verificado experimentalmente, diferindo, contudo, quanto aos valores da força de ruptura. A força determinada com o modelo analítico foi igual a $665,07 \mathrm{kN}$, obtendo-se um erro de 16,87 \% em comparação com o resultado experimental, sendo a ruína do bloco ocorrida devido à ruptura da escora junto ao pilar. Essa diferença maior em contraste com os modelos numéricos pode ser explicada devido ao modelo analítico prever certa folga de resistência para as escoras.

\section{Conclusões}

A modelagem proposta, utilizando o Método dos Elementos Finitos e os modelos constitutivos simplificados, apresentou razoável desempenho em confronto com o resultado experimental e com o numérico obtido por Souza (2004) quanto à força de ruptura e ao panorama de fissuração no concreto. A ruína do bloco sobre estacas foi prevista pelo modelo proposto com um erro relativo de $0,62 \%$.

No que concerne à análise mecânica de blocos sobre estacas, verifica-se que a utilização de modelo constitutivo baseado na Mecânica do Dano Contínuo para simular o comportamento do concreto constitui uma válida alternativa. No entanto, a aplicação conjunta de tal modelo em situações práticas fica prejudicada em função da identificação experimental dos parâmetros contidos na lei de evolução da variável de dano.

Considerou-se que os materiais concreto e aço tivessem perfeita aderência no modelo estrutural; entretanto, melhores resultados podem ser conseguidos por meio da implementação de um modelo de aderência entre as barras de armadura e o concreto (como, por exemplo, o indicado pelo Código Modelo CEB-FIP 1990 (1993)), 
possibilitando assim prever os deslizamentos relativos e as tensões de aderência na interface.

A modelagem numérica proposta conseguiu predizer a distribuição de fissuras no concreto do bloco sobre duas estacas. Essa metodologia apresenta a vantagem de não requerer o uso de técnicas de reconstrução da malha de elementos finitos. Em geral, modelos discretos necessitam de técnicas sofisticadas e onerosas computacionalmente de reconstrução da malha para capturar a propagação de fissuras ao longo do processo de carga.

Análises numéricas com outros tipos de elementos finitos de maior ordem e refinamento de malha são necessárias, de maneira a realizar um estudo da influência da malha (convergência) na resposta do modelo quanto à distribuição de dano na estrutura - possibilidade de surgimento de novas fissuras.

Nas simulações efetuadas, pode-se considerar que as estacas estivessem apoiadas em material indeformável, uma vez que translações na direção y foram restringidas. Como sugestão de pesquisa futura, análises numéricas do bloco considerando as estacas apoiadas sobre material deformável (por meio de molas dotadas de modelo constitutivo não linear, por exemplo) podem ser realizadas.

\section{Referências}

BLÉVOT, J.; FRÉMY, R. Semelles sur pieux. Annales d'Institut Technique du Bâtiment et des Travaux Publics, Paris, v. 20, n. 230, p. 223-295, 1967.

COMITÉ EURO-INTERNATIONAL DU BETON - CEB. Fip Model Code 1990. Bulletin d'Information, Lausanne, n. 213/214, p. 33-41, 1993.

GUELLO, G. A.; BITTENCOURT, T. N. Simulação computacional de estruturas de concreto por meio da mecânica do dano. Boletim Técnico da Escola Politécnica da USP, v. 0202, n. 1, p. 1-12, 2002.
KRENK, S.; HEDEDAL, O. A dual ortogonality procedure for nonlinear finite element equations. Comput. Methods Appl. Mech. Engrg., v. 123, p. 95-107, 1995.

MAUTONI, M. Blocos sobre dois apoios. São Paulo: Grêmio Politécnico, Escola Politécnica da Universidade de São Paulo, 1972.

MAZARS, J. Aplication de la mécanique de l'endommagement au comportament non lineare et à la rupture du béton de structure. 1984. Thése (Doctorat d'État) - Université Paris 6, Paris.

MORAES, M. C. Estruturas de fundações. São Paulo: McGraw-Hill, 1976.

MUNHOZ, F. S. Análise do comportamento de blocos de concreto armado sobre estacas submetidos à ação de força centrada. 2004. Dissertação (Mestrado em Engenharia de Estruturas) - Departamento de Engenharia, Universidade de São Paulo, São Carlos.

OLIVEIRA, D. S.; BARROS, S.; GIONGO, J. S. Blocos de concreto armado sobre seis estacas: simulação numérica e dimensionamento pelo método de bielas e tirantes. Revista IBRACON de Estruturas e Materiais, v. 7, n. 1, p. 1-23, 2014.

PITUBA, J. J. C.; PROENÇA, S. P. B. Estudo e aplicação de modelos constitutivos para o concreto fundamentados na mecânica do dano contínuo. Cadernos de Engenharia de Estruturas, v. 7, n. 23, p. 33-60, 2005.

RAMM, E. Strategies for tracing the nonlinear response near limit points. In: EURO-USWORSHOP ON NONLINEAR FINITE ELEMENT ANALYSIS IN STRUCTURAL MECHANICS, 1981, Berlin. Proceedings... Ruhr Universitat Bochum, p. 68-89, Springer, Berlin, 1981.

SOUZA, L. A. F.; MACHADO, R. D. Análise numérico-computacional de estruturas de concreto armado considerando o dano, a fratura e critério de falha. Revista IBRACON de Estruturas e Materiais, v. 6, p. 101-120, 2013. 
SOUZA, R. A. Concreto estrutural: análise e dimensionamento de elementos com descontinuidades. 2004. Tese (Doutorado) - Escola Politécnica, Universidade de São Paulo, São Paulo.

SOUZA, R. A.; BITTENCOURT, T. N. Análise não - linear de bloco rígido sobre duas estacas. Engenharia Civil UM (Braga), Universidade do Minho, v. 21, p. 25-38, 2004.

- Aplicação do Método das Bielas. Téchne $\overline{R e v i s t a}$ de Tecnologia da Construção, São Paulo, v. 97, p. 56-60, 2005.

WESSELS, M. Das statische und dynamishe durchschlagsproblem der imperfektem flachen kugelchale bei elasticher rotatiossymmetrischer verformumg. 1977. Dissertation T. U. Hannover, Mitteil, n. 23 des Imstituts für Statik.

YANG, Y. B.; KUO, S. B. Theory \& analysis of nonlinear framed structures. New York: Prentice Hall, 1994. 